\section{Letalidade hospitalar nas angioplastias coronárias no Estado do Rio de Janeiro, Brasil, 1999-2003}

\author{
Hospital case fatality associated with coronary \\ angioplasty in Rio de Janeiro State, Brazil, \\ 1999-2003
}

\author{
${ }^{1}$ Faculdade de Medicina, \\ Universidade Federal do \\ Rio de Janeiro, \\ Rio de Janeiro, Brasil. \\ 2 Escola Nacional de Saúde \\ Pública Sergio Arouca, \\ Fundação Oswaldo Cruz, \\ Rio de Janeiro, Brasil. \\ 3 Secretaria de Estado da \\ Saúde do Estado do \\ Rio de Janeiro, \\ Rio de Janeiro, Brasil. \\ Correspondência \\ P. H. Godoy \\ Faculdade de Medicina, \\ Universidade Federal do \\ Rio de Janeiro. \\ Rua Dr. Satamini 183, apto. 601 , \\ Rio de Janeiro, $R J$ \\ 20270-233, Brasil. \\ phgodoy@infolink.com.br
}

\begin{abstract}
This study analyzes hospital case fatality associated with percutaneous transluminal coronary angioplasties (PTCA) covered by the Brazilian Unified National Health System (SUS) and performed in hospitals in the State of Rio de Janeiro from 1999 to 2003. PTCA data were obtained from the State Health Department's database on Authorizations for Hospital Admissions. Case fatality rates were estimated according to age, gender, diagnosis, and hospital. Overall case fatality was $1.9 \%$ in 8,735 PTCAs. The lowest rate was associated with angina (0.8\%) and the highest rates with acute myocardial infarction (6\%) and other diagnoses (7\%). In the 50-69-year bracket, case fatality was higher in women. In the over70 group, it was almost three times that of the youngest group (4\% versus $1.4 \%$ ). There was great variability among PTCA case fatality rates in different hospitals (from 0 to 6.5\%). Ongoing monitoring of PTCAs is thus necessary in clinical practice. In conclusion, PTCA performance was still unsatisfactory under the Unified $\mathrm{Na}$ tional Health System.
\end{abstract}

Angioplasty; Myocardial Ischemia; Lethality
Paulo Henrique Godoy 1 Carlos Henrique Klein 2

Nelson Albuquerque de Souza-e-Silva 1,3 Gláucia Maria Moraes de Oliveira 1

\section{Introdução}

A angioplastia coronária vem sendo utilizada cada vez mais como procedimento para revascularização do miocárdio. A angioplastia coronária foi realizada pela primeira vez em 1977 por Andreas Gruentzig, nos Estados Unidos, e no Brasil foi introduzida no final de 19791.

No mundo ocidental, são executados cerca de 400 mil procedimentos por ano, que representam cerca de 40 a $50 \%$ do total dos procedimentos de revascularização miocárdica ${ }^{2}$. Nos Estados Unidos 3, em 1999, foram realizados mais de um milhão de procedimentos de angioplastias coronárias, e no Brasil, de 2000 a 2004, perto de 24,5 mil angioplastias coronárias por ano, segundo o Departamento de Informática do SUS (DATASUS: http://datasus.gov.br, acessado em 24/Jul/2005).

Face à crescente utilização da angioplastia coronária nas doenças isquêmicas do coração, encontram-se na literatura estudos sobre sua efetividade clínica e custo, comparação com trombolíticos ${ }^{4}$ e com cirurgia de revascularização do miocárdio ${ }^{3}$. Os escores de risco, como o CADILLAC risk score ${ }^{5}$, calculados para predizer a mortalidade pós-angioplastia coronária primária no infarto agudo do miocárdio, corroboram a necessidade de estudos sobre o procedimento.

Este estudo objetiva estimar as taxas de letalidade, segundo sexo, faixas etárias, grupos de diagnóstico e hospitais, nas internações em que 
foram realizadas angioplastia coronária pagas pelo Sistema Único de Saúde (SUS), no Estado do Rio de Janeiro, nos anos de 1999 a 2003.

\section{Material e métodos}

As informações sobre as angioplastias coronárias foram obtidas nos bancos de dados do Sistema de Informações Hospitalares do Sistema Único de Saúde (SIH/SUS), que compreendem as Autorizações de Internação Hospitalar (AIH) pagas no Estado do Rio de Janeiro, referentes ao período de 1999 a 2003. Os dados foram fornecidos pela Secretaria de Estado de Saúde do Estado do Rio de Janeiro (SES-RJ).

Os procedimentos de angioplastia coronária estudados foram os que receberam os códigos 32023014 (angioplastia coronariana) e 32035012 (coronarioplastia para implante duplo de prótese), segundo o SIH/SUS.

Os grupos diagnósticos de doenças isquêmicas do coração foram organizados com a utilização dos códigos da 10a conferência de revisão da Classificação Internacional de Doenças (CID-10) 6, da seguinte forma: infarto agudo do miocárdio (I21 a I23), anginas (I20), outras doenças isquêmicas agudas do coração (I24) e doenças isquêmicas crônicas do coração (I25). Foi incluído também um conjunto complementar de outros diagnósticos, com códigos não relacionados nos grupos de doenças isquêmicas do coração, em que foram realizadas angioplastias coronárias. Isso porque se pressupõe a existência de doenças isquêmicas do coração sempre que se realiza angioplastia coronária.

Os hospitais vinculados ao SUS, identificados apenas com letras, foram individualizados desde que tivessem realizado pelo menos duzentas angioplastias coronárias no período. De 14 hospitais que realizaram angioplastia coronária no período estudado, 12 preencheram este critério; os dois restantes foram agrupados numa categoria denominada de "Demais".

As taxas de letalidade foram estimadas segundo faixas etárias ( 20 a 49, 50 a 69, e 70 anos ou mais), sexo, grupos de diagnósticos e hospitais. As taxas de letalidade por hospital foram também ajustadas segundo idade (em anos completos), sexo e grupos de diagnósticos, pela regressão de Poisson 7. Foi utilizado o programa Stata, versão 7 (Stata Corporation, College Station, Estados Unidos).

Este estudo teve seu projeto submetido ao Comitê de Ética em Pesquisa da Universidade Federal do Rio de Janeiro, sendo apreciado e aprovado em 14 de julho de 2005, tendo o protocolo de pesquisa o número 102/05.

\section{Resultados}

Foram realizadas 8.735 angioplastias coronárias no Estado do Rio de Janeiro, de 1999 a 2003. Nos grupos diagnósticos, 4.913 (56,2\%) estavam relacionadas às doenças isquêmicas agudas do miocárdio (infarto agudo do miocárdio, anginas e outras isquemias agudas), 3.565 (40,8\%) às doenças isquêmicas crônicas, e 257 (2,9\%) aos outros diagnósticos, sem menção à presença de doenças isquêmicas do coração (Tabela 1). As angioplastias coronárias foram menos freqüentes quando associadas com outros diagnósticos ou infarto agudo do miocárdio, e nestes grupos ocorreram as taxas de letalidade mais elevadas, de 7 e $6 \%$, respectivamente. A menor letalidade, de $0,8 \%$, foi observada no grupo das anginas (Tabela 1).

Observaram-se flutuações nas taxas de letalidade ao longo dos anos estudados, tanto no total dos grupos diagnósticos como em cada um. No total, ocorreu crescimento da letalidade até o ano de 2001 e, a partir daí, queda até o menor valor da série no último ano, de 2003 (1,4\%). A letalidade geral no período foi de $1,9 \%$ (Tabela 1 ).

Durante o período estudado, ocorreu uma queda nítida na freqüência da associação de outros diagnósticos, sem menção a doenças isquêmicas do coração, como as angioplastias coronárias. Nas isquêmicas crônicas, as taxas de letalidade nas angioplastias coronárias apresentaram tendência de queda ao longo dos anos. Já nas isquêmicas agudas tal tendência não se manifestou, e no grupo mais freqüente, das anginas, ocorreu até mesmo elevação das taxas de letalidade e do número de angioplastias coronárias ao longo do período (Tabela 1).

A idade nas angioplastias coronárias variou de 20 a 96 anos. A taxa de letalidade nas angioplastias coronárias foi discretamente maior nas mulheres. A letalidade nas mulheres só foi menor do que nos homens na faixa etária de 50 a 69 anos. Nesta faixa etária, a letalidade feminina também foi menor do que nas de 20 a 49 anos. Ressalta-se o aumento relevante da taxa de letalidade dos de 70 anos ou mais, de pelo menos três vezes em relação aos mais jovens, em ambos os sexos, em qualquer grupo diagnóstico. Nos grupos de isquêmicas agudas as taxas de letalidade foram mais elevadas nos homens, enquanto nas isquêmicas crônicas prevaleceram as taxas das mulheres (Tabela 2).

Nos hospitais as taxas brutas de letalidade variaram de 0 a $6,5 \%$, e as ajustadas de 0 a $2,8 \%$. As taxas brutas mais baixas parecem ter se concentrado nos hospitais com maiores volumes de procedimentos. O coeficiente correlação linear de Pearson entre estas taxas de letalidade, brutas, e os volumes foi de -0,35, entretanto o mesmo co- 
Taxas de letalidade nas internações por angioplastia coronária segundo os grupos de diagnósticos e os anos, nos hospitais do Estado do Rio de Janeiro, Brasil, 1999-2003.

\begin{tabular}{|c|c|c|c|c|c|c|c|c|c|c|c|c|}
\hline \multirow[t]{2}{*}{ Grupos de diagnósticos } & \multicolumn{2}{|c|}{1999} & \multicolumn{2}{|c|}{2000} & \multicolumn{2}{|c|}{2001} & \multicolumn{2}{|c|}{2002} & \multicolumn{2}{|c|}{2003} & \multicolumn{2}{|c|}{ Total } \\
\hline & $\%$ & n & $\%$ & n & $\%$ & n & $\%$ & n & $\%$ & n & $\%$ & n \\
\hline Infarto agudo do miocárdio & 3,9 & 233 & 3,5 & 114 & 13,6 & 66 & 9,3 & 140 & 5,2 & 250 & 6,0 & 803 \\
\hline Anginas & 0,2 & 528 & 0,6 & 157 & 0,0 & 428 & 1,2 & 747 & 1,3 & 1.117 & 0,8 & 2.977 \\
\hline Outras isquêmicas agudas & 0,0 & 41 & 0,5 & 206 & 1,4 & 288 & 1,6 & 321 & 0,7 & 277 & 1,1 & 1.133 \\
\hline Doenças isquêmicas crônicas & 3,2 & 187 & 1,8 & 874 & 2,4 & 956 & 1,4 & 844 & 0,7 & 704 & 1,7 & 3.565 \\
\hline Outros diagnósticos & 2,3 & 43 & 8,3 & 121 & 6,3 & 79 & 14,3 & 14 & - & - & 7,0 & 257 \\
\hline Total & 1,6 & 1.302 & 2,2 & 1.472 & 2,3 & 1.817 & 2,0 & 2.066 & 1,4 & 2.348 & 1,9 & 8.735 \\
\hline
\end{tabular}

Fonte: Sistema de Informações Hospitalares do Sistema Único de Saúde.

Tabela 2

Taxas de letalidade nas angioplastias coronárias, por 100 internações pagas pelo SUS, segundo grupos etários, grupos de diagnóstico e sexo. Estado do Rio de Janeiro, Brasil, 1999-2003.

\begin{tabular}{|c|c|c|c|c|c|c|c|c|c|c|c|c|c|c|c|c|c|c|c|c|c|c|c|c|}
\hline \multirow{4}{*}{$\begin{array}{l}\text { Grupos } \\
\text { etários } \\
\text { (anos) }\end{array}$} & \multicolumn{24}{|c|}{ Grupos de diagnósticos e sexo } \\
\hline & \multicolumn{4}{|c|}{$\begin{array}{l}\text { Infarto agudo } \\
\text { do miocárdio }\end{array}$} & \multicolumn{4}{|c|}{ Anginas } & \multicolumn{4}{|c|}{$\begin{array}{l}\text { Outras } \\
\text { isquêmicas } \\
\text { agudas }\end{array}$} & \multicolumn{4}{|c|}{$\begin{array}{l}\text { Doenças } \\
\text { isquêmicas } \\
\text { crônicas }\end{array}$} & \multicolumn{4}{|c|}{$\begin{array}{c}\text { Outros } \\
\text { diagnósticos }\end{array}$} & \multicolumn{4}{|c|}{ Total } \\
\hline & \multicolumn{2}{|c|}{ Feminino } & \multicolumn{2}{|c|}{ Masculino } & \multicolumn{2}{|c|}{ Feminino } & \multicolumn{2}{|c|}{ Masculino } & \multicolumn{2}{|c|}{ Feminino } & \multicolumn{2}{|c|}{ Masculino } & \multicolumn{2}{|c|}{ Feminino } & \multicolumn{2}{|c|}{ Masculino } & \multicolumn{2}{|c|}{ Feminino } & \multicolumn{2}{|c|}{ Masculino } & \multicolumn{2}{|c|}{ Feminino } & \multicolumn{2}{|c|}{ Masculino } \\
\hline & $\%$ & $\mathbf{n}$ & $\%$ & $n$ & $\%$ & $\mathrm{n}$ & $\%$ & $n$ & $\%$ & $n$ & $\%$ & $\mathrm{n}$ & $\%$ & n & $\%$ & n & $\%$ & $\mathrm{n}$ & $\%$ & $\mathbf{n}$ & $\%$ & $n$ & $\%$ & $n$ \\
\hline $20-49$ & 0,0 & 48 & 1,8 & 114 & 0,6 & 173 & 0,3 & 371 & 0,0 & 59 & 0,0 & 134 & 1,5 & 198 & 1,3 & 456 & 18,8 & 16 & 2,3 & 43 & 1,4 & 494 & 0,9 & 1.118 \\
\hline $50-69$ & 5,7 & 158 & 6,4 & 326 & 0,3 & 634 & 0,4 & 1.182 & 0,8 & 245 & 1,3 & 452 & 1,1 & 727 & 1,2 & 1.447 & 0,0 & 43 & 5,8 & 104 & 1,2 & 1.807 & 1,6 & 3.511 \\
\hline$\geq 70$ & 9,5 & 74 & 10,8 & 83 & 1,9 & 265 & 3,1 & 352 & 1,9 & 108 & 1,5 & 135 & 4,4 & 293 & 3,4 & 444 & 21,7 & 23 & 10,7 & 28 & 4,2 & 763 & 3,9 & 1.042 \\
\hline Total & 5,7 & 280 & 6,1 & 523 & 0,7 & 1.072 & 0,9 & 1.905 & 1,0 & 412 & 1,1 & 721 & 2,0 & 1.218 & 1,6 & 2.347 & 9,8 & 82 & 5,7 & 175 & 2,0 & 3.064 & 1,9 & 5.671 \\
\hline
\end{tabular}

eficiente substituindo-se as taxas brutas de letalidade pelas ajustadas foi de 0,14 . Assim, apesar de parecer que nos hospitais com maior volume a letalidade tenha sido menor, a relação letalidadevolume se reduziu consideravelmente quando se fez o ajuste que levou em conta os efeitos de sexo, idade e grupos diagnósticos (Tabela 3).

O hospital C, o que mais realizou angioplastias coronárias nos infartos agudos do miocárdio, apresentou a menor taxa de letalidade. Apenas cinco hospitais, de um total de 14, A, B, F, G e J, realizaram $98,5 \%$ das angioplastias coronárias no grupo das doenças isquêmicas crônicas. Nestes, o subconjunto dos hospitais A e B, que são públicos, apresentou letalidade sete vezes menor do que o subconjunto formado por F, G e J, que são privados. No grupo das anginas $77,5 \%$ das angioplastias coronárias foram feitas nos hospitais A,
E, J e K. O subconjunto formado pelos hospitais A, E e K apresentou letalidade 9,5 vezes menor do que o hospital J nas anginas (Tabela 3).

\section{Discussão}

A Organização para o Desenvolvimento e Cooperação Econômica (OECD: http://www.oecd. org, acessado em 20/Jun/2005), que agrega trinta países membros, a maioria européia, mantendo relações com setenta outros países, publica estatísticas econômicas e sociais, incluindo saúde. Os dados da OECD sobre doenças isquêmicas do coração relativos ao período de 1999 a 2003 revelam que a taxa de realização de angioplastia coronária mais elevada ocorreu nos Estados Unidos, de 381,1 e 426,3, por 100 mil habitantes, 
Taxas de letalidade nas angioplastias coronárias, por 100 internações pagas pelo SUS, segundo hospitais selecionados e grupos diagnósticos, brutas e ajustadas *. Estado do Rio de Janeiro, Brasil, 1999-2003.

\begin{tabular}{|c|c|c|c|c|c|c|c|c|c|c|c|c|c|}
\hline \multirow[t]{5}{*}{ Hospitais ** } & \multicolumn{10}{|c|}{ Grupos de diagnósticos } & \multicolumn{3}{|c|}{ Total } \\
\hline & \multirow{3}{*}{\multicolumn{2}{|c|}{$\begin{array}{l}\text { Infarto } \\
\text { agudo do } \\
\text { miocárdio }\end{array}$}} & \multirow{3}{*}{\multicolumn{2}{|c|}{ Anginas }} & \multirow{3}{*}{\multicolumn{2}{|c|}{$\begin{array}{l}\text { Outras } \\
\text { isquêmicas } \\
\text { agudas }\end{array}$}} & \multirow{3}{*}{\multicolumn{2}{|c|}{$\begin{array}{l}\text { Doenças } \\
\text { isquêmicas } \\
\text { crônicas }\end{array}$}} & \multirow{3}{*}{\multicolumn{2}{|c|}{$\begin{array}{c}\text { Outros } \\
\text { diagnósticos }\end{array}$}} & \multirow{3}{*}{\multicolumn{2}{|c|}{ Brutas }} & \multirow[t]{3}{*}{ Ajustadas } \\
\hline & & & & & & & & & & & & & \\
\hline & & & & & & & & & & & & & \\
\hline & $\%$ & n & $\%$ & $\mathrm{n}$ & $\%$ & $\mathbf{n}$ & $\%$ & $\mathbf{n}$ & $\%$ & $\mathrm{n}$ & $\%$ & $\mathrm{n}$ & $\%$ \\
\hline A & 7,1 & 85 & 0,5 & 631 & 0,0 & 270 & 0,2 & 404 & 0,0 & 4 & 0,7 & 1.394 & 0,6 \\
\hline$B$ & 9,8 & 51 & 0,3 & 312 & & & 0,4 & 499 & 12,5 & 8 & 1,0 & 870 & 0,9 \\
\hline C & 1,4 & 286 & 0,0 & 1 & 1,9 & 470 & & & 0,0 & 1 & 1,7 & 758 & 0,6 \\
\hline D & 7,8 & 51 & 1,6 & 125 & 33,3 & 3 & 0,0 & 7 & 20,0 & 15 & 5,0 & 201 & 2,2 \\
\hline$E$ & & & 0,0 & 504 & & & & & 0,0 & 6 & 0,0 & 510 & 0,0 \\
\hline $\mathrm{F}$ & & & & & & & 2,3 & 427 & & & 2,3 & 427 & 2,8 \\
\hline G & 18,2 & 11 & 0,0 & 2 & & & 2,2 & 1.707 & & & 2,3 & 1.720 & 2,7 \\
\hline $\mathrm{H}$ & 10,1 & 79 & 0,0 & 2 & 0,0 & 167 & 0,0 & 2 & 13,6 & 22 & 4,0 & 272 & 1,6 \\
\hline I & 10,9 & 64 & 4,3 & 47 & 0,6 & 160 & 25,0 & 4 & 6,1 & 180 & 4,8 & 455 & 1,5 \\
\hline$J$ & 0,0 & 1 & 1,9 & 583 & & & 2,1 & 474 & 0,0 & 4 & 2,0 & 1.062 & 1,9 \\
\hline K & & & 0,0 & 588 & & & & & & & 0,0 & 588 & 0,0 \\
\hline O & 7,4 & 162 & 5,0 & 121 & 0,0 & 2 & 16,7 & 6 & & & 6,5 & 291 & 2,0 \\
\hline Demais & 0,0 & 13 & 0,0 & 61 & 1,6 & 61 & 0,0 & 35 & 0,0 & 17 & 0,5 & 187 & 0,0 \\
\hline Total & 6,0 & 803 & 0,8 & 2.977 & 1,1 & 1.133 & 1,7 & 3.565 & 7,0 & 257 & 1,9 & 8.735 & 1,9 \\
\hline
\end{tabular}

* Modelo Poisson, por idade, sexo e grupos de diagnósticos;

** A a O, hospitais com 200 ou mais angioplastias coronárias realizadas; Demais, com menos de 200 angioplastias coronárias realizadas.

no início e no final deste período, respectivamente. Os Estados Unidos foram seguidos pela Alemanha com taxas de 202,4 e 269,8, por $100 \mathrm{mil}$ habitantes, nos mesmos anos. As taxas mais baixas de realização de angioplastia coronária, nos países monitorados pela OECD, ocorreram no México, que em 1999 apresentou taxa de 0,8 por 100 mil habitantes e, em 2003, de 1,4. Portugal veio a seguir com taxas de 40,5 e 66,1, por $100 \mathrm{mil}$ habitantes, respectivamente. No Estado do Rio de Janeiro as taxas de realização de angioplastias coronárias pagas pelo SUS foram de 7,5 e 15,5 por 100 mil habitantes, em 1999 e 2003, respectivamente. Seguramente as taxas de realização totais de angioplastias coronárias no Estado do Rio de Janeiro devem ter sido maiores já que deveriam incluir aquelas feitas no sistema privado, porém não se conhecem os dados desta esfera de forma abrangente.

A Central Nacional de Investigações Cardiovasculares (CENIC), órgão da Sociedade Brasileira de Hemodinâmica e Cardiologia Intervencionista (SBHCI: http://www.sbhci.org.br, acessado em 07/Abr/2005), foi criada em 1991 com a finalidade de documentar o desempenho e a evolução da especialidade no Brasil. O banco de dados da CENIC é alimentado por meio da contribuição voluntária dos sócios autorizados para a reali- zação dos procedimentos percutâneos, sem discriminar intervenções pagas pelo SUS ou por seguros de saúde ou por particulares. Contudo, os dados da CENIC são incompletos porque registram 25.505 angioplastias coronárias no Brasil, em 2003, quando só o DATASUS relata que foram pagas pelo SUS 30.666 angioplastias coronárias no mesmo ano.

O presente estudo analisa alguns dados contidos nas AIH somente sobre as angioplastias coronárias pagas pelo SUS, deve-se, entretanto, considerar que a qualidade dessas informações não é constante, uma vez que, se são confiáveis os registros de realização de angioplastias coronárias e de óbitos associados, os critérios utilizados para registrar os diagnósticos podem variar de forma relevante entre instituições e profissionais. Ressalta-se que não há registros de ensaios clínicos brasileiros sobre angioplastia coronária que possam permitir comparações com os resultados obtidos neste estudo. O CENIC apresenta taxas de letalidade nas angioplastias coronárias para o total do país que variaram de 1,28 a $0,73 \%$ de 1999 a 2003, sem infarto agudo do miocárdio. No Estado do Rio de Janeiro, a taxa de letalidade nas angioplastias coronárias pagas pelo SUS, excluindo os infartos agudos do miocárdio, foi de $1 \%$, semelhante, naqueles mesmos anos de 
1999 e 2003. No entanto o CENIC não discrimina as taxas por estados, impedindo a comparação direta com os dados do SUS no Estado do Rio de Janeiro. E, ainda mais, também é possível supor que exista viés de seleção das informações pela incompletude dos dados do CENIC, fato que pode distorcer a avaliação de desempenho.

No período de 1999 a 2003 foram pagas pelo SUS cerca de 4,7 milhões de internações no Estado do Rio de Janeiro, das quais 68.375 com diagnóstico de doenças isquêmicas do coração. Estas internações se elevaram de 12,2 mil, em 1999, para 14,5 mil, em 2003. A quantidade de procedimentos de alta complexidade inerentes às doenças isquêmicas do coração (angioplastia coronária e revascularização do miocárdio, os mais freqüentes), pagos pelo SUS, também aumentou no período: de 2.974 para 4.892 , o que representa um aumento de $64,5 \%$ a mais. Porém, enquanto nas revascularizações do miocárdio foi verificado um decréscimo de 35\% (1.041) para $23 \%$ (1.125) dos procedimentos de alta complexidade, as angioplastias coronárias cresceram de $35 \%$ (1.041) para 48\% (2.348), ou seja, tiveram um incremento de $12 \%$ na sua participação relativa no volume dos procedimentos de alta complexidade pagos pelo SUS no Estado do Rio de Janeiro. O incremento da realização das angioplastias coronárias no Estado do Rio de Janeiro também foi observado na maioria dos países cobertos pela OECD, com crescimento de até $254 \%$, entre 1990 e 2000 .

Neste estudo, a avaliação da eficácia dos procedimentos de angioplastia coronária se restringe à análise das taxas de letalidade no período de internação. E, ainda é preciso ressaltar que foram utilizados como denominadores das taxas de letalidade as internações e não os indivíduos, o que significa que as taxas de letalidade por indivíduos devem ser ainda maiores.

A taxa de letalidade nas angioplastias coronárias variou de acordo com o diagnóstico associado. As maiores taxas ocorreram nos infartos agudos do miocárdio e nos outros diagnósticos, sem menção a doenças isquêmicas do coração, como esperado, visto que nos infartos agudos do miocárdio devem ocorrer procedimentos primários, vale dizer, não eletivos, enquanto $80 \%$ dos diagnósticos sem menção a doenças isquêmicas do coração foram compostos por insuficiências cardíacas, que pressupõem pacientes com pior prognóstico geral. Nos diagnósticos de doenças isquêmicas do coração crônicas, poder-se-ia esperar que a taxa de letalidade nas angioplastias coronárias fosse menor do que nos grupos das anginas e das outras isquêmicas agudas, por estarem associadas, com maior probabilidade, aos procedimentos eletivos. Todavia, a letalidade neste grupo foi mais do que duas vezes superior àquela verificada nas anginas. É possível especular que pacientes com pior prognóstico, com função ventricular deteriorada, estivessem no grupo das doenças isquêmicas do coração crônicas, mas também é possível que informações enviesadas sobre diagnósticos tenham distorcido estes resultados. Por sinal, nas doenças isquêmicas do coração crônicas tanto a letalidade quanto o volume de diagnósticos reduziram-se depois de 2001, ao contrário do que ocorreu com as anginas, em que a letalidade e o volume cresceram depois daquele ano, mostrando provavelmente $o$ viés de informações diagnósticas (Tabela 1).

Também como nas internações com revascularização do miocárdio ${ }^{8}$, nas angioplastias coronárias, cerca de dois terços foram de pacientes do sexo masculino, semelhante ao que se verificou na distribuição da mortalidade por cardiopatia isquêmica na população em geral 9 . Estudo de coortes retrospectivas, baseadas em informações de prontuários, realizado por Passos et al. 10, constatou que as taxas de letalidade de homens e mulheres com infarto agudo do miocárdio, submetidos à angioplastia coronária primária, foram marcadamente similares: 14,6 e $14,3 \%$, respectivamente. Em nosso estudo a taxa de letalidade no infarto agudo do miocárdio foi um pouco maior nos homens $(6,1 \%)$ do que nas mulheres (5,7\%). Em relação à idade, o que chama mais atenção é a taxa de letalidade nas internações nos pacientes de setenta anos ou mais, que chegou a ser pelo menos três vezes maior do que nos mais jovens, em ambos os sexos e em qualquer grupo diagnóstico (Tabela 2).

Os resultados do TIME trial 11 não revelaram diferenças significativas no que se refere à morte, a sintomas, à qualidade de vida e ao infarto não fatal, quando comparadas as estratégias invasivas de revascularização com terapêuticas medicamentosas otimizadas em pacientes idosos (com oitenta anos de idade, em média) com doença arterial crônica sintomática, após seguimento de um ano. Em estudo de pacientes tratados na Mayo Clinic, Estados Unidos, envolvendo 1.597 pessoas, entre as quais 127 com oitenta anos ou mais, a mortalidade associada com angioplastia primária no infarto agudo do miocárdio manteve-se alta entre octogenários 12 . Tais achados devem levar à reflexão sobre o benefício do procedimento em pacientes mais idosos. Em ensaio clínico randomizado 13 , a letalidade anual de pacientes com angina estável e doença de um ou dois vasos tratados clinicamente foi menor do que $2,3 \%$, e em outro estudo recente 14 , pacientes que tinham doença isquêmica coronariana estável, com ou sem infarto agudo do miocárdio prévio apresentaram letalidade anual com o tra- 
tamento clínico otimizado de apenas 0,8\%. Estes resultados podem levar ao questionamento da indicação indiscriminada da angioplastia coronária com o objetivo de reduzir a mortalidade cardiovascular. Quando o desempenho imediato da angioplastia coronária medido pela letalidade hospitalar estiver próxima a $4 \%$, como ocorreu nos pacientes de setenta anos ou mais no presente estudo (Tabela 2), torna-se precária a justificativa para a realização de angioplastia coronária com o objetivo de reduzir a letalidade nos idosos com doenças isquêmicas do coração. Tendo como base nossos resultados e os de outros estudos 15 , a angioplastia coronária poderia ser reservada para aliviar os sintomas de pacientes com angina estável, caso o tratamento clínico não alcance esse objetivo, ou ainda, para casos especiais nos quais as drogas do tratamento clínico otimizado não possam ser utilizadas.

Quanto aos hospitais, observou-se grande variabilidade entre as taxas de letalidade nas instituições, e em relação às taxas brutas houve relação inversa entre volume anual de procedimentos e letalidade, uma vez que as taxas brutas mais altas se concentraram nos hospitais com menor número de procedimentos. Contudo, a relação letalidade-volume praticamente desapareceu quando se ajustaram as taxas, levando em consideração sexo, idade e grupos diagnósticos.

O hospital C chama atenção pela taxa de letalidade nos infartos agudos do miocárdio, comparada à dos outros hospitais (Tabela 3). Todos aqueles hospitais com quantidades razoáveis, de mais de cinqüenta angioplastias coronárias no período, neste grupo diagnóstico, tiveram letalidades variando entre 7,1 e $10,9 \%$, enquanto a taxa no hospital C foi de 1,4\%. Se retirássemos o referido hospital do total, a taxa de letalidade geral para angioplastia coronária no infarto agudo do miocárdio aumentaria de 6 para 8,5\%. Ainda no hospital C, se removêssemos aquelas angioplastias coronárias associadas ao infarto agudo do miocárdio, a taxa de letalidade aumentaria de 1,7 para 1,9\%. É possível supor que erros na atribuição ou registro dos diagnósticos tenham distorcido os resultados no hospital C, podendo estar inseridas neste contexto síndromes coronarianas crônicas internadas para procedimentos eletivos e não para angioplastia coronária primária, visto que este hospital tem setor de emergência sem acesso direto de pacientes e tem processo de internação que deixa poucas possibilidades para receber pacientes com menos de noventa minutos após início do quadro clínico de infarto agudo do miocárdio.

Frente a estes resultados, assim como aos de outros trabalhos citados, o crescente uso das angioplastias coronárias, pagas pelo SUS, tornase necessário acompanhar de modo contínuo a adequação de seu uso. É preciso verificar, de forma permanente, se o que inicialmente parecia ser útil quando esta tecnologia foi implantada e disseminada no sistema de saúde, com base no desempenho esperado segundo os resultados de alguns ensaios terapêuticos, foi ou não confirmado pela prática clínica. Para isto, não basta apenas interpretar os resultados dos ensaios clínicos que comparam técnicas, como a revascularização do miocárdio e a angioplastia coronária, entre si ou contra os resultados do tratamento clínico. É preciso incorporar a idéia de "performance mínima", entendida como aquela que é eficaz não apenas nos ensaios clínicos, mas também na sua aplicação na assistência aos pacientes 16. Em conclusão, a performance da angioplastia coronária no âmbito da modalidade de atenção pelo SUS nos hospitais, dentro do período estudado, não foi satisfatória.

\section{Resumo}

O estudo analisa a letalidade hospitalar nas angioplastias coronárias, pagas pelo Sistema Único de Saúde (SUS), realizadas nos hospitais do Estado do Rio de Janeiro, Brasil, de 1999 até 2003. As informações sobre as angioplastias coronárias provieram do banco de Autorizações de Internações Hospitalares da Secretaria de Estado de Saúde do Estado do Rio de Janeiro. As taxas de letalidade foram estimadas segundo faixas etárias, sexo, diagnósticos e hospitais. A letalidade geral foi de 1,9\% em 8.735 angioplastias coronárias. A taxa mais baixa ocorreu nas anginas (0,8\%), as mais elevadas nos infartos agudos do miocárdio (6\%) e em outros diagnósticos (7\%). A letalidade foi menor nas mulheres na faixa etária entre 50 e 69 anos, e a partir dos setenta anos foi quase três vezes maior que a dos mais jovens (de 1,4 a 4\%), em ambos os sexos. Ocorreu grande variabilidade entre as taxas de letalidade nas angioplastias coronárias nos diferentes hospitais (entre 0 e 6,5\%). Portanto, é necessário acompanhar de modo contínuo a adequação da utilização da angioplastia coronária. Em conclusão, a performance deste procedimento no âmbito da modalidade de atenção pelo SUS nos hospitais, dentro do período estudado, não foi satisfatória.

Angioplastia; Isquemia Miocárdica; Letalidade 


\section{Colaboradores}

P. H. Godoy participou do planejamento, da execução e da análise do estudo, e ainda foi responsável pelo manuscrito e levantamento bibliográfico. C. H. Klein, N. A Souza-e-Silva e G. M. M. Oliveira participaram de todas as fases do estudo e da revisão do manuscrito.

\section{Agradecimentos}

Ao José Luiz Zedane e à Ângela Maria Cascão pela colaboração imprescindível no acesso aos bancos de dados da Secretaria de Estado da Saúde do Estado do Rio de Janeiro.

\section{Referências}

1. Sousa JEMR, Buchler JR, Pimentel Filho WA, Moraes AG. Angioplastia coronária transluminal percutânea. Arq Bras Cardiol 1980; 35:1-4.

2. Sociedade Brasileira de Cardiologia. Diretrizes da Sociedade Brasileira de Cardiologia sobre angioplastia transluminal coronária. Arq Bras Cardiol 1995; 64:491-500.

3. Hoffman SN, TenBrook JA, Wolf MP, Pauker SG, Salem DN, Wong JB. A meta-analysis of randomized controlled trials comparing coronary artery bypass graft with percutaneous transluminal coronary angioplasty: one- to eight-year outcomes. J Am Coll Cardiol 2003; 41:1293-304.

4. Hartwell D, Colquitt J, Loveman E, Clegg AJ, Brodin $\mathrm{H}$, Waugh N, et al. Clinical effectiveness and costeffectiveness of immediate angioplasty for acute myocardial infarction: systematic review and economic evaluation. Health Technol Assess 2005; 9:1-99.

5. Halkin A, Singh M, Nikolsky E, Grines CL, Tcheng JE, Garcia E, et al. Prediction of mortality after primary percutaneous coronary intervention for acute myocardial infarction: the CADILLAC risk score. J Am Coll Cardiol 2005; 45:1397-405.

6. Organização Mundial da Saúde. Classificação estatística internacional de doenças e problemas relacionados à saúde. 10a Revisão. São Paulo: Edusp; 1995.

7. Kleinbaum DG, Kupper LL, Miller KE, Nizam A. Applied regression analysis and multivariable methods. 3rd Ed. Pacific Grove: Duxbury Press; 1998.

8. Godoy PH, Klein CH, Souza-e-Silva NA, Oliveira GMM, Fonseca TMP. Letalidade na cirurgia de revascularização do miocárdio no Estado do Rio de Janeiro - SIH/SUS - no período 1999-2003. Rev SOCERJ 2005; 18:23-9.

9. Oliveira GMM. Mortalidade cardiovascular no Estado do Rio de Janeiro no período de 1980 a 2000 [Tese de Doutorado]. Rio de Janeiro: Universidade Federal do Rio de Janeiro; 2003.
10. Passos LCS, Lopes AA, Esteves FA, Santos FMO. Diferença de letalidade hospitalar do infarto agudo do miocárdio entre homens e mulheres submetidos à angioplastia primária. Arq Bras Cardiol 1998; 71:587-90.

11. Pfisterer M, Buser P, Osswald S, Allemann U, Amann W, Angehrn W, et al. Outcome of elderly patients with chronic symptomatic coronary artery disease with an invasive vs. optimized medical treatment strategy: one-year results of the randomized TIME trial. JAMA 2003; 289:1117-23.

12. Singh M, Mathew V, Garratt KN, Berger PB, Grill DE, Bell MR, et al. Effect of age on the outcome of angioplasty for acute myocardial infarction among patients treated at the Mayo Clinic. Am J Med 2000; 108:187-92.

13. Yusuf S, Zucker D, Peduzzi P, Fisher LD, Takaro T, Kennedy JW, et al. Effect of coronary artery bypass graft surgery on survival: overview of 10-year results from randomised trials by the Coronary $\mathrm{Ar}-$ tery Bypass Graft Surgery Trialists Collaboration. Lancet 1994; 344:563-70.

14. Jabbour S, Young-Xu Y, Graboys TB, Blatt CM, Goldberg RJ, Bedell SE, et al. Long-term outcomes of optimized medical management of outpatients with stable coronary artery disease. Am J Cardiol 2004; 93:294-9.

15. Rihal CS, Raco DL, Gersh BJ, Yusuf S. Indications for coronary artery bypass surgery and percutaneous coronary intervention in chronic stable angina: review of the evidence and methodological considerations. Circulation 2003; 108:2439-45.

16. Souza-e-Silva NA. Performance e tecnologia em medicina. O caso da revascularização miocárdica por cirurgia ou por angioplastia. Rev SOCERJ 2005; 18:123-30.

Recebido em 18/Nov/2005

Versão final reapresentada em 29/Jun/2006 Aprovado em 01/Ago/2006 